\title{
Hemodynamic, ventilatory and gasometric evaluation of an experimental bronchopleural fistula ${ }^{1}$
}

\author{
Douglas Guimaraes de Oliveira ${ }^{\text {I }}$, Maria Gabriela Cavicchia Toneloto'II, Marcos Mello Moreira' ${ }^{\text {III }}$, Joaquim Murray Bustorff-Silva ${ }^{\text {IV }}$, \\ Gabriel Franco de Souzav, Luiz Claudio Martins" ${ }^{\text {VI }}$, Carolina Kosour ${ }^{\text {VII }}$, Desanka Dragosavac ${ }^{\text {VIII }}$, Antonio Luis Eiras Falcão ${ }^{\text {VIII }}$
}

\author{
DOI: http://dx.doi.org/10.1590/S0102-86502015001000001
}

IFellow Master Degree, Postgraduate Program in Surgery Sciences, Department of Surgery, Faculty of Medical Sciences, State University of Campinas (UNICAMP), Brazil. Intellectual and scientific content, design of the study; technical procedures; manuscript writing; supervised all phases of the study. IIPhD, Full Professor, Division of Pneumology, Department of Physical Therapy, University of Jaguariuna, Brazil. Design of the study, technical procedures, manuscript writing, supervised all phases of the study.

IIIPhD, Division of Pneumology, Department of Internal Medicine, Visitor Professor of Department of Surgery, Medical School, UNICAMP, CampinasSP, Brazil. Acquisition and interpretation of data, design the study, technical procedures.

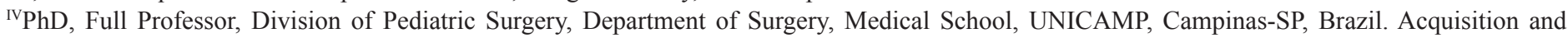
interpretation of data, technical procedures, English version.

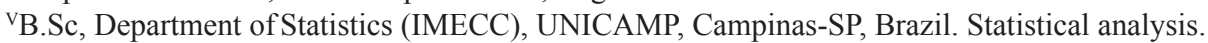

VIPhD, Physician Assistant, Division of Physiology and Metabology in Surgery, Department of Surgery, Medical School, UNICAMP, Campinas-SP, Brazil. Acquisition and interpretation of data, design the study, involved with technical procedures.

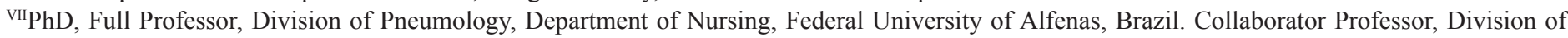
Physiology and Metabology in Surgery, Department of Surgery, Medical School, UNICAMP, Campinas-SP, Brazil. Design the study, acquisition and interpretation of data, technical procedures.

VIIIPhD, Full Professor, Division of Physiology and Metabology in Surgery, Department of Surgery, Medical School, UNICAMP, Campinas-SP, Brazil. Design the study, acquisition and interpretation of data, technical procedures.

\section{ABSTRACT}

PURPOSE: To investigate the hemodynamic and ventilatory changes associated with the creation of an experimental bronchopleural fistula (BPF) treated by mechanical ventilation and thoracic drainage with or without a water seal.

METHODS: Six large white pigs weighing $25 \mathrm{~kg}$ each which, after general anesthesia, underwent endotracheal intubation (6mm), and mechanically ventilation. Through a left thoracotomy, a resection of the lingula was performed in order to create a BPF with an output exceeding $50 \%$ of the inspired volume. The chest cavity was closed and drained into the water sealed system for initial observation of the high output BPF.

RESULTS: Significant reduction in BPF output and $\mathrm{PaCO}_{2}$ was related after insertion of a water-sealed thoracic drain, $\mathrm{p}<0.05$.

CONCLUSION: Insertion of a water-sealed thoracic drain resulted in reduction in bronchopleural fistula output and better $\mathrm{CO}_{2}$ clearance without any drop in cardiac output or significant changes in mean arterial pressure.

Key words: Respiratory Tract Fistula. Respiration, Artificial. Models, Animal. Swine. 


\section{Introduction}

Bronchopleural fistulas (BPFs) are a frequent cause of complications in the ICU, being associated with high morbidity and mortality, especially when secondary to invasive mechanical ventilation. It manifests initially as a tension pneumothorax that, after drainage, results in persistent air leak. The diagnosis is established when the drainage bottle shows continuous bubbling for more than $24 \mathrm{hs}^{1-10}$.

Among its several etiologies the most important are pneumonia, radiotherapy, tumours, tuberculosis, lung surgeries, trauma and, inside ICU's it is frequently associated to prolonged positive pressure ventilation and barotrauma. Regardless of its etiology, BPFs are very hard to treat, mainly because most of the patients will need mechanical ventilation to keep their oxygenation status stable and this in turn will tend to hamper the closure of the BPF. When the air leak is severe morbidity is very high and mortality can reach $18 \%$ up to $50 \%{ }^{10-16}$.

Successful management depends on the strategies used, that should be tailored to the individual patient. Zimmerman et $a .^{7}$, recommended removing mechanical ventilation and especially PEEP (positive end expiratory pressure) to treat BPFs but it is feared that this might result in severe hypoxia. Downs and Chapman $^{8}$, achieved cessation of air leak during expiration by applying positive intra-pleural pressure equivalent to the PEEP. However a small amount of air leak persisted during inspiration and also, this procedure resulted in a significant incidence of alveolar collapse and pneumothorax ${ }^{9,10}$. The alternative strategy of aspirating the drainage bottle, although efficient in reducing the pneumothorax, may result in increased air leak which might further compromise the ventilatory status of the patient $t^{1,3,12}$.

Other authors have described methods of selective occlusion of the thoracic drain during different periods of the respiratory cycle reporting varying results. The great variety of therapeutic strategies reported, up to now, to treat BPFs, points to the need of a better understanding of the changes in ventilatory mechanics and respiratory status associated to the presence of the air leak $\mathrm{k}^{3,6,17-20}$.

Accordingly, the purpose of this paper is to describe the hemodynamic and ventilatory changes associated with the creation of an experimental BPF treated by mechanical ventilation (MV) and thoracic drainage with or without a water seal.

\section{Methods}

This study was approved by the Ethics Committee on Animal Experimentation of the Institute of Biology, State University of Campinas (Protocol No. 1685-1 A).
Six large white pigs were used weighing $25 \mathrm{~kg}$ each which, after general anesthesia, underwent endotracheal intubation $(6 \mathrm{~mm})$, and mechanically ventilation. The endotracheal tube and the mechanical ventilator circuit were connected through the sensor of volumetric capnography $\left(\mathrm{CO}_{2} \mathrm{SMO}\right.$ Plus DX-8100 ${ }^{\circledR}$ Dixtal/Novametrix), which was calibrated before the beginning of each experiment. The software used to capture and store data online and offline enabled the measurement (in $\mathrm{mL}$ ) of BPF output, which was calculated by subtracting the expired volume (Ve) from the inspired volume (Vi). The percentage of fistula output was defined as the result of this subtraction ( Vi $\mathrm{Ve})$ divided by the Vi.

A pulmonary artery catheter (PAC) was introduced through the femoral vein. Mean arterial pressure (MAP) was recorded continuously (Multiparameter Monitor Dixtal DX-2020; AJIBP-0). Data from arterial and venous blood gases and hemooximetry were collected before (baseline), after creation of BPF and after each experimental setting.

The animals received a continuous infusion of saline $(0.9 \%)$ as well as anesthetics, sedatives and neuromuscular blockers as needed.

\section{Surgical procedure}

Through a left thoracotomy, a resection of the lingula was performed in order to create a BPF with an output exceeding $50 \%$ of the inspired volume. The chest cavity was closed and drained into the water sealed system for initial observation of the high output BPF (Figure 1).

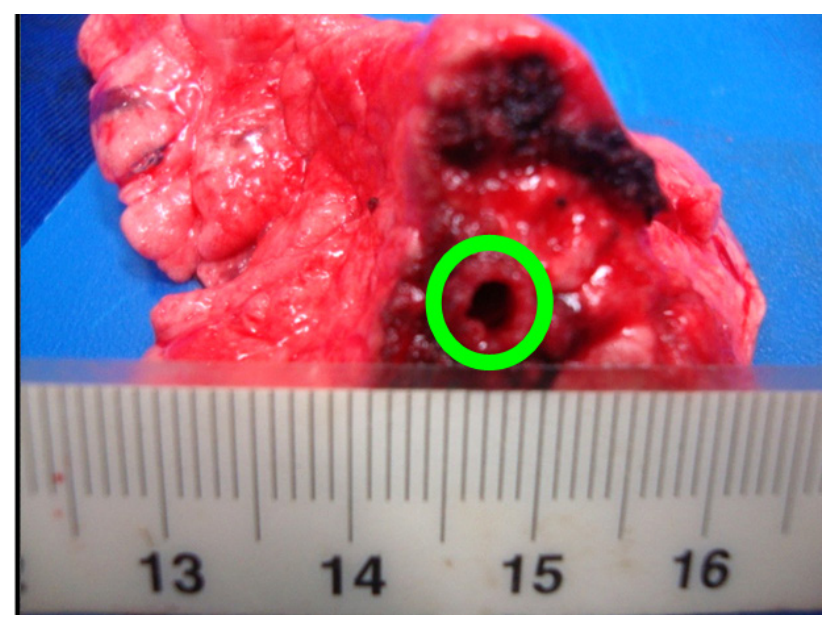

FIGURE 1 - Light bronchus (green circle) with approximately four millimeters in diameter. 


\section{Experimental protocol of mechanical ventilation}

The MV protocol consisted of Volume Controlled Ventilation $(\mathrm{VCV})$, with a Tidal Volume $\left(\mathrm{V}_{\mathrm{T}}\right)$ of $8 \mathrm{ml} / \mathrm{Kg}$, Fraction of Inspired Oxygen $\left(\mathrm{FiO}_{2}\right)$ of 0.21 , Inspiratory time versus Expiratory time $(\mathrm{I}: \mathrm{E}$ ratio) $=1: 2$, Respiratory Rate $(\mathrm{RR})$ of 22 bpm, Positive End Expiratory Pressure (PEEP) of $5 \mathrm{cmH}_{2} \mathrm{O}$. Using these ventilatory parameters, it was possible to maintain the end-tidal $\mathrm{CO}_{2}\left(\mathrm{PetCO}_{2}\right)$ around $45 \mathrm{mmHg}$.

\section{Statistical analysis}

Comparison of the hemodynamic and ventilatory parameters among the different phases of the experiment was performed using repeated measures ANOVA, considering each pig as an experimental block. Differences were considered significant when $\mathrm{p}<0.05$.

\section{Results}

The results of changes in ventilation and hemodynamic changes recorded in pre-fistula (baseline) and after the creation of the BPF (simple drainage and water seal) are arranged in Table 1.

TABLE 1 - Data of volumetric capnography, blood gas analysis and hemodynamic expressed as mean and standard deviation.

\begin{tabular}{ccccc}
\hline & $\begin{array}{c}\text { Pre-fistula } \\
\text { (basal) }\end{array}$ & Open Drain & Water seal & p \\
\hline BPFO & - & $63 \pm 16.2$ & $42.3 \pm 10.8$ & 0.0443 \\
PetCO $_{2}$ & $43.2 \pm 1.8$ & $43.2 \pm 21.5$ & $51.0 \pm 9.5$ & 0.5686 \\
PaCO $_{2}$ & $46 \pm 3.8$ & $61.3 \pm 10.0$ & $54.6 \pm 12.3$ & 0.0303 \\
VT alv & $173.5 \pm 34.9$ & $60.6 \pm 15$ & $92 \pm 25.7$ & 0.0596 \\
PaO $_{2}$ & $79.5 \pm 11.9$ & $54.4 \pm 18.4$ & $63.3 \pm 21.3$ & 0.2304 \\
SatO $_{2}$ & $88.6 \pm 7.1$ & $57.9 \pm 22.3$ & $68.9 \pm 23.9$ & 0.2392 \\
Raw & $14.4 \pm 5.9$ & $44 \pm 13.2$ & $39.6 \pm 15.8$ & 0.2817 \\
PIT & $20.5 \pm 4.2$ & $17.5 \pm 6.6$ & $21.0 \pm 4.5$ & 0.1941 \\
MAP & $86.7 \pm 17.6$ & $119.8 \pm 24.7$ & $128.2 \pm 20.5$ & 0.3368 \\
CO & $3.7 \pm 0.8$ & $4.3 \pm 0.6$ & $4.9 \pm 1.6$ & 0.3495 \\
\hline
\end{tabular}

BPFO (\%): Bronchopleural fistula output; $\mathrm{PetCO}_{2}(\mathrm{mmHg})$ : pressure of end-tidal $\mathrm{CO}_{2} ; \mathrm{PaCO}_{2}(\mathrm{mmHg})$ : partial pressure of $\mathrm{CO}_{2}$ in arterial blood; $\mathrm{V}_{\mathrm{T}}$ alv $(\mathrm{ml})$ : alveolar tidal volume; $\mathrm{PaO}_{2}(\mathrm{mmHg})$ : partial pressure of $\mathrm{O}_{2} ; \mathrm{Sat}_{2} \mathrm{O}_{2}(\%)$ : oxygen saturation in arterial blood; $\mathrm{Raw}\left(\mathrm{cmH}_{2} \mathrm{O} / \mathrm{L} / \mathrm{s}\right)$ : resistance airway: PIT $\left(\mathrm{cmH}_{2} \mathrm{O}\right)$ : intrathoracic pressure; MAP $(\mathrm{mmHg})$ : mean arterial pressure; $\mathrm{CO}(\mathrm{L} / \mathrm{min})$ : cardiac output.

As it may be observed in this table, significant differences between the phases were observed only in the BPFO and $\mathrm{PaCO}_{2}$ values. Although alveolar ventilation showed a slight improvement with the installation of water sealed drainage this difference did not attained statistical significance (Figure 2).

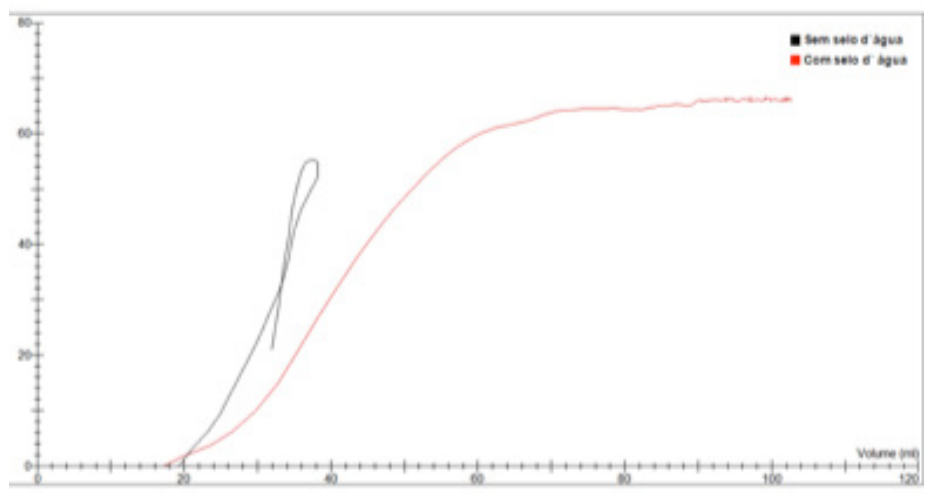

FIGURE 2 - Volumetric Capnography x Treatments. In the "x" axis, tidal volume $(\mathrm{mL})$ and the " $\mathrm{y}$ " axis, the $\mathrm{CO}_{2}$ partial pressure $(\mathrm{mmHg})$. Note that alveolar volume behaves better during the water seal treatment (red line).

Below is the representative model of volumetric capnography curves during their treatments.

\section{Discussion}

Despite the need to reduce morbidity and mortality of BPF therapy, particularly when associated with mechanical ventilation, little has been advanced in this field in the last decades. Several studies have described tentative strategies to control BPF output during $\mathrm{MV}^{1-9}$. However, these studies are mostly case reports and reviews, which failed to present an adequate solution to complications such as pneumothorax, hemodynamic instability, persistent air leak and severe hypoxemia ${ }^{8-12,16}$. This demonstrates the heterogeneous nature of the problem and partially explains the non-standardization of therapy in BPF. This study proposes a physiologic analysis of the consequences of the creation of a BPF in an experimental setting.

Several strategies have been tried to treat patients with high output BPF needing MV. Shekar et al. ${ }^{4}$ maintained the airway pressure below the opening pressure of the BPF, and then optimized the pleural suction pressure thus preventing aggravations. Additionally, Kempainem and Pierson ${ }^{3}$, considered the use of low tidal volume and increased inspiratory/expiratory ratio, as a protective strategy of FBP under VM, because it reduces the peak pressure and avoids auto-PEEP. These authors proposed, in order to reduce the output of the FBP, the use of minimal levels of expiratory pressure, short inspiratory time, low tidal volume and low frequency.

As expected, in the present experiment, there was a progressive increase in $\mathrm{PaCO}_{2}$ and inspiratory resistance after the induction of the BPF, both of which improved after the 
introduction of water-sealed thoracic drainage. In some cases, increasing the ventilatory rate might compensate the air leak, thus preventing or at least reducing the $\mathrm{CO}_{2}$ retention. However, this could increase mean airway pressure, reduce cardiac output and increase fistula output. A slight, not statically significant increase of the PIT was observed during our experiment (data not shown).

In order to mechanically reduce the BPF output, Gallagher et al. ${ }^{13}$, proposed the insertion of a unidirectional valve in the drainage bottle, synchronized with the inspiratory phase of the mechanical ventilator. This procedure did not produce any deleterious effect over the cardiopulmonary function. In the present experiment, insertion of a water-sealed thoracic drain resulted in improved saturation and better $\mathrm{CO}_{2}$ clearance without any drop in cardiac output or significant changes in MAP. Analysis of volumetric capnography data from Figure 2 confirms that these changes resulted in a slight improvement of alveolar ventilation, although this improvement was not statically significant. Although this study is limited by the fact that it is an experimental study and that the experiments were performed on previously healthy lungs, the combined analysis of these data allows us to recommend water-sealed drainage as an efficient strategy to treat BPF. However, in order to reduce morbidity and mortality associated with this condition, it is of utmost importance that both surgical and non-surgical techniques to promote early closures of these fistulas continue to be pursued.

\section{Conclusion}

Insertion of a water-sealed thoracic drain resulted in reduction in bronchopleural fistula output and better $\mathrm{CO}_{2}$ clearance without any drop in cardiac output or significant changes in mean arterial pressure.

\section{References}

1. Pierson DJ, Horton CA, Bates PW. Persistent bronchopleural air leak during mechanical ventilation. Chest. 1986 Sep;90(3):321-3. doi:10.1378/chest.90.3.321.

2. Bishop MJ, Benson MS, Sato P, Pierson DJ. Comparison of highfrequency jet ventilation with conventional mechanical ventilation for bronchopleural fistula. Anesth Analg. 1987 Sep;66:833-8. PMID: 3304022.

3. Kempainen RR, Pierson DJ. Persistent air leaks in patients receiving mechanical ventilation. Semin Respir Crit Care Med. 2001 Dec;22(6):675-84. PMID: 16088712.

4. Shekar K, Foot C, Fraser J, Ziegenfuss M, Hopkins P,Windsor M. Bronchopleural fistula: an update for intensivists. J Crit Care. 2010 Mar;25:47-55. doi: 10.1016/j.jcrc.2009.05.007.
5. Baumann M, Sahn S. Medical management and therapy of bronchopleural fistulas in the mechanically ventilated patient. Chest. 1990 Mar;97:721-8. PMID: 2407455.

6. Martins WR, Siefkin AP, Allen R. Closure of a bronchopleural fistula with bronchoscopic instillation of tetracycline. Chest. 1991 Apr;99:1040-2. PMID: 2009764.

7. Zimmerman JE, Colgan DL, Mills M. Management of bronchopleural fistula complicating therapy with positive end expiratory pressure (PEEP). Chest. 1973;64(4):526-9. PMID: 4582828.

8. Downs JB, Chapman RL. Treatment of bronchopleural fistula during continuous positive pressure ventilation. Chest. 1976;69(3):363-6. PMID: 786563.

9. Powner DJ, Grenvik A. Ventilatory management of life-threatening bronchopleural fistulae: a summary. Crit Care Med. 1981;9(1):54-8. PMID: 7006912.

10. Phillips YY, Lonigan RM Joyner LR. A simple technique for managing a bronchopleural fistula while maintaining positive pressure ventilation. Crit Care Med. 1979;7(8):351-3. PMID: 378544.

11. Pruit RF, Messick WJ, Thomason MH. Respiratory alkalosis caused by assist control mechanical ventilation in a patient with a bronchopleural fistula. J Trauma. 1996;40(3):481-2. PMID: 8601875

12. Sager JS, Eiger G, Fuchs BD. Ventilator auto-triggering in a patient with tuberculous bronchopleural fistula. Resp Care. 2003;48(5):51921. PMID: 12729469.

13. Gallagher TJ, Smith AR, Kirby RR, Civetta JM. Intermittent inspiratory chest tube occlusion to limit bronchopleural cutaneous airleaks. Crit Care Med. 1976;4(6):328-32. PMID: 803091.

14. Litmamovitch M, Joynt GM, Bates PW. Persistent bronchopleural fistula in a patient with adult respiratory distress syndrome. Treatment with pressure-controlled ventilation. Chest. 1993;104(6):1901-2. PMID: 8252982.

15. Bevelaqua FA, Kay S. A modified technique for the management of bronchopleural fistula in ventilator - dependent patients: a report of two cases. Respir Care. 1986 Feb;31(10):904-8. PMID: 2493107.

16. Sarkar P, Chandak T, Shah R, Talwar A. Diagnosis and management bronchopleural fistula. Indian J Chest Dis Allied Sci. 2010 AprJun;52(2):97-104. PMID: 20578402.

17. Andreetti C, D'Andrilli A, Ibrahim M, Ciccone AM, Maurizi G, Mattia A, Venuta F, Rendina EA. Effective treatment of postpneumonectomy bronchopleural fistual by conical fully covered self-expandable stent. Interact Cardiovasc Thorac Surg. 2012 Apr;14(4):420-3. PMID: 22268070.

18. Wang H, Li D, Zhang N, Zou H, Luo L, Ma H, Zhou Y, Li J, Liang S. Sealing of airway fistulas for metallic covered z-type stents. Zhongguo Fei Ai Za Zhi. 2011 Aug;14(8):679-84. doi: 10.3779/j. issn.1009-3419.2011.08.08.

19. Leo F, Solli P, Veronesi G, Galetta D, Petrella F, Gasparri R, Borri A, Spaggiari L. Review on bronchopleural fistula. Did a surgeon review it? Chest. 2006 Jun;129:1731-2. PMID: 16778298.

20. Maung AA, Kaplan LJ. Mechanical ventilation after injury. J Intensive Care Med. 2012 May-Jun29(3):128-37. doi: 10.1177/0885066612457339.

\section{Acknowledgements}

Center for Experimental Medicine and Surgery, Faculty of Medical Sciences, UNICAMP, especially to biologists Willian Adalberto Silva and Ana Cristina Moraes. 


\section{Correspondence:}

Antônio Luís Eiras Falcão

Hospital de Clínicas - UNICAMP

Rua Vital Brasil, 251

Cidade Universitária Zeferino Vaz

13083-888 Campinas - SP Brasil

aefalcao@gmail.com

Received: Sep 19, 2014

Review: Nov 17, 2014

Accepted: Dec 18, 2014

Conflict of interest: none

Financial source: São Paulo Research Foundation (Process 04610-2/2009)

${ }^{1}$ Research performed at Center for Experimental Medicine and Surgery, Faculty of Medical Sciences, State University of Campinas (UNICAMP), Brazil. 